\title{
Parameters estimation of systems with delayed and structured entries
}

\author{
Lotfi Belkoura ${ }^{\mathrm{a}}$ Jean-Pierre Richard ${ }^{\mathrm{b}}$ Michel Fliess ${ }^{\mathrm{c}}$ \\ ${ }^{\mathrm{a}}$ LAGIS (CNRS, UMR 8146) \& Alien Project (INRIA Lille-Nord Europe), \\ Université des Sciences et Technologies de Lille, France \\ (e-mail: lotfi.belkoura@univ-lille1.fr) \\ ${ }^{\mathrm{b}}$ LAGIS (CNRS, UMR 8146) \& Alien Project (INRIA Lille-Nord Europe), \\ École Centrale de Lille, France, \\ (e-mail: jean-pierre.richard@ec-lille.fr) \\ ${ }^{\mathrm{c}}$ LIX (CNRS, UMR 7161) E Alien Project (INRIA Saclay Ile de France), \\ École Polytechnique, France \\ (e-mail: Michel.Fliess@polytechnique.edu)
}

\begin{abstract}
This paper deals with on-line identification of continuous-time systems with structured entries. Such entries, which may consist in inputs, perturbations or piecewise polynomial (time varying) parameters, can be defined as signals that can be easily annihilated. The proposed cancellation method allows to obtain non asymptotic estimators for the unknown coefficients. Application to delayed and switching hybrid systems are proposed. Numerical simulations with noisy data but also experimental results on a delay process are provided.
\end{abstract}

Key words: Delay systems, Switched linear systems, Identification.

\section{Introduction}

System identification deals with the problem of building mathematical models of dynamical systems based on observed data. Even though considerable effort have been undertaken on various aspects of parameters estimation, there are still many open problems due to the specific structure of the underlying models. Particularly, estimation of continuous time systems with delay phenomenon $[17,18,4,5]$ or switching systems $[12,20]$ have received particular attention during the last few years, although we find generally no common approach in dealing with delayed and hybrid systems.

This paper dwells on the fast identification techniques (non asymptotic) initially proposed by M. Fliess \& H. Sira-Ramirez [11] in the framework of finite dimensional models. While most of the existing estimation techniques have been done in a probabilistic setting, this approach presents a new standpoint based on differential algebra, module theory and operational calculus, which was a most classical tool among control engineers. Several successful applications and laboratory experiments have already been performed (see e.g; [6] for applications to nonlinear state estimation, $[7,8]$ for linear and nonlinear diagnosis, and [10,16] for signal processing). The contribution of this paper mainly concerns the extension of the former technique to systems with structured entries (inputs or parameters), and for which retarded phenomena occur. It constitutes an extension of [4], and, as in $[2,3,24]$, the results are stated within the distribution framework, although some of them will be also stated using the operational calculus.

The retarded phenomena we shall be respectively concerned with are those due to delayed inputs or step changes in the parameters. Hence, while [4] only considers linear time delay systems, this paper not only generalizes the approach to possibly nonlinear processes, but also shows how the presented technique can provide new estimation tools for the specific class of time varying (switching) systems.

Among the existing techniques for delay estimations, and regardless of the shift-operator technique of the popular Padé type (which limits are depicted in [19], par- 
ticularly for control design and time-varying delays), $[13,17]$ suggest an adaptive procedure based on Lyapunov Krasovskii functionals where the delays are assumed to be known or in a fixed discrete set. In their illustrative examples, more than $100 \mathrm{sec}$. are required for a significant estimation of delays not exceeding $1 \mathrm{sec}$. Also asymptotic, the least-squares based technique proposed in [18] suffers from the same poor rate of convergence (more than $120 \mathrm{sec}$ of convergence for estimating a delay value of $1.3 \mathrm{sec}$.). Also, off-line procedures based on linear filters are given in [1], where piece-wise constant inputs are assumed and additional iterative steps are required to deal with non linearity in the unknown delay. For both delay and switching parameters estimation problems, the novelty and efficiency of the proposed identification algorithms mainly lie in their non asymptotic nature, therefore enlarging the scope and potentialities of real time control procedures.

The paper is organized as follows. Section 2 states the definition of structured terms we shall use in the sequel. Their annihilation, the structure of the underlying estimation problem, and the design of causal relations from input and output derivatives are presented. Sections 3 and 4 are devoted respectively to applications to delayed and switched linear systems identification. Simulations and experiments illustrate the results and exhibit convergence times of the order 1 to 10 times the delays. Most of the results are performed using a distributional standpoint and the usual definitions and basic properties are given below.

\section{General Framework}

We recall here some standard definitions and results from distribution theory [22], and fix the notations to be used in the sequel. The space of $C^{\infty}$-functions having compact support in an open subset $\Omega$ of $\mathbb{R}$ is denoted by $\mathcal{D}(\Omega)$, and $\mathcal{D}^{\prime}(\Omega)$ is the space of distributions on $\Omega$, i.e., the space of continuous linear functionals on $\mathcal{D}(\Omega)$.

For $T \in \mathcal{D}^{\prime},\langle T, \varphi\rangle$ denotes the real number which linearly and continuously depends on $\varphi \in \mathcal{D}$. This number is defined as $\langle T, \varphi\rangle=\int_{-\infty}^{\infty} f . \varphi$ for a locally bounded function $T=f$. For the Dirac distribution $T=\delta$ and its derivative $T=\dot{\delta}$, the functional is defined as $\langle T, \varphi\rangle=$ $\varphi(0)$ and $\langle T, \varphi\rangle=\dot{\varphi}(0)$ respectively.

The complement of the largest open subset of $\Omega$ in which a distribution $T$ vanishes is called the support of $T$ and is written $\operatorname{supp} T$. Write $\mathcal{D}_{+}^{\prime}\left(\right.$ resp. $\left.\mathcal{E}^{\prime}\right)$ the space of distributions with support contained in $[0, \infty)$ (resp. compact support). It is an algebra with respect to convolution with identity $\delta$, the Dirac distribution. For $T, S \in$ $\mathcal{D}_{+}^{\prime}$, the convolution product is defined as $\langle T * S, \varphi\rangle=$ $\langle T(x) . S(y), \varphi(x+y)\rangle$, and can be identified with the familiar convolution product $(T * S)(t)=\int_{-\infty}^{\infty} T(\theta) S(t-$ $\theta) d \theta$ in case of locally bounded functions $T$ and $S$.
All the distributions we shall consider in the sequel are assumed to belong to $\mathcal{D}_{+}^{\prime}$. When concentrated at a point $\{\tau\}$, the Dirac distribution $\delta(t-\tau)$ is written $\delta_{\tau}$. A distribution is said to be of order $r$ if it acts continuously on $C^{r}$-functions but not on $C^{r-1}$-functions. Measures and functions are of order 0 . Functions are considered through the distributions they define and are therefore indefinitely differentiable. Hence, if $y$ is a continuous function except at a point $a$ with a finite jump $\sigma_{a}$, its derivative writes $\dot{y}=d y / d t+\sigma_{a} \delta_{a}$, where $d y / d t$ is the distribution stemming from the usual derivative of $y$. Derivation, integration and translation can be formed from the convolution products $\dot{y}=\delta^{(1)} * y, \quad \int y=$ $H * y, \quad y(t-\tau)=\delta_{\tau} * y$, where $\delta^{(1)}($ or $\dot{\delta})$ is the derivative of the Dirac distribution, and $H$ is the familiar Heaviside step function. For $S, T \in \mathcal{D}_{+}^{\prime}$, supp $S * T \subset$ supp $S+\operatorname{supp} T$, where the sum in the right hand side is defined by $\{x+y ; x \in \operatorname{supp} S, y \in \operatorname{supp} T\}$. Finally, with no danger of confusion, we shall denote $T(s), s \in$ $\mathbb{C}$, the Laplace transform of $T$.

\section{Annihilation of structured entries and Inte- gration by part formulas}

\subsection{Structured entries: definition and annihilation}

Structured entries have been introduced (see e.g. [11,23]) to refer to entities (mainly perturbations) that can be annihilated by means of simple multiplications and derivations. A simple example consists for instance in a load perturbation, modeled by a Heaviside function $\xi(t)=$ $H(t)$, and for which one easily obtains, in the time and operational domain respectively:

$$
t \times \frac{d \xi}{d t}=0, \quad \frac{d}{d s} s \xi(s)=0
$$

In this context, a perturbation entry $\xi$ is said to be structured if the following module is a torsion module:

$$
\operatorname{span}_{k\left[t, \frac{d}{d t}\right]}(\xi)=0
$$

where $k\left[t, \frac{d}{d t}\right]$ is the ring of differential operators with polynomial coefficients. We adopt in this paper an alternative statement in which multiplication is not restricted to polynomials of $t$, but may be extended to exponential functions. In some applications, this extension may avoid multiplication of noisy data with unbounded (polynomial) terms. Moreover, the term "structured" will not only refer to perturbations but may also concern initial conditions, inputs or time varying parameters. For a formal definition, we may state that a distribution $\xi$ is said to be structured if one can write

$$
P * \xi=Q
$$


for some $P \in \mathcal{E}^{\prime}$ and $Q \in \mathcal{D}_{+}^{\prime}$, both with discrete support. This statement is sufficient for most of the practical situations (and more), and includes piecewise polynomials, exponential and harmonic functions, impulsive functions and theirs derivatives (Dirac functions used to take into account initial conditions in the distribution framework) etc. For the sake of completeness, let us remark that a counter example can be found in smooth functions with compact support, whose convolution with any distribution $P$ results in a smooth right hand side (r.h.s.) $Q$. Such definition allows, at least formally, a large choice in the candidate function for multiplication since, by virtue of Schwartz Theorem [22], $\alpha Q=0$ for any smooth function $\alpha$ whose derivatives of appropriate order vanish on the support of $Q$, yielding the annihilation:

$$
\alpha \times(P * \xi)=0 .
$$

Note that with the statement (3), the candidate function $\alpha$ only depends on the structure of $Q$. If $Q$ admits a decomposition of the form $Q=\sum Q_{i}$, where the $Q_{i}$ have disjoint supports, then its annihilation requires the product $\alpha=\Pi \alpha_{i}$. Situations of practical interest are those for which the two following points hold.

- The structured term $\xi$ is involved in an equation of the form:

$$
R * \omega=\xi,
$$

where $R$ is a (possibly delayed) differential operator, and $\omega$ is a vector of (functions of) measurements. Combined with (4), this leads to the measurement based relation:

$$
\alpha \times(P * R * \omega)=0 .
$$

- The candidate function $\alpha$ is chosen as a linear combination of polynomial and/or exponential terms. This choice allows to turn product of the form $t^{p} y^{(q)}$ (resp. $\left.e^{-\gamma t} y^{(q)}\right)$ into exact differentiations of the form: $\left(t^{k} y\right)^{q}$ (resp. $\left.\left(e^{-\gamma t} y\right)^{q}\right)$.

These aspects are discussed in the subsequent paragraphs:

\subsection{Structure of the estimation problem}

Annihilating a structured term $\xi$ does not necessarily imply that all informations about this term are lost. Depending on the coefficients to be estimated, different structures for the estimation problem may occur. Throughout this paper, the structure of the model is known a priori and particularly:

- The process is assumed to be linear with respect to its unknown parameters (but not necessarily w.r.t. to its inputs/outputs),

- Inputs or time varying parameters from which delays or switching times are to be estimated consist in structured signal (piecewise constant for most practical cases) as described in the previous section.
The estimation procedure is based on the idea that unknown terms to be identified and contained in a structured term can be recovered by the above cancellation procedure.

The following example of a second order system subject to nonzero initial condition and delayed step inputs illustrates this observation. Formulated respectively in the operational (Laplace transform) and distributional framework, let us consider the system governed by:

$$
\begin{aligned}
& s^{2} y+a_{1} s y+a_{0} y=s y_{0}+\dot{y}_{0}+u_{1} \frac{e^{-\tau_{1} s}}{s}+u_{2} \frac{e^{-\tau_{2} s}}{s} \\
& \ddot{y}+a_{1} \dot{y}+a_{0} y=y_{0} \dot{\delta}+\dot{y}_{0} \delta+u_{1} H_{\tau_{1}}+u_{2} H_{\tau_{2}} .
\end{aligned}
$$

The structured term $\xi$ consists here in the right hand side member of these equations and the system is described by equation (5) with $R=\left(\ddot{\delta}+a_{1} \dot{\delta}+a_{0} \delta\right)$, and $\omega=y$. In turn $\xi$ satisfies (3) with $P=\dot{\delta}$ and $Q=\ddot{\delta} y_{0}+\dot{\delta} \dot{y}_{0}+$ $u_{1} \delta_{\tau_{1}}+u_{2} \delta_{\tau_{2}}$, since one may write:

$$
\begin{aligned}
& s \xi=s^{2} y_{0}+s \dot{y}_{0}+u_{1} e^{-\tau_{1} s}+u_{2} e^{-\tau_{2} s}, \\
& \dot{\xi}=\ddot{\delta} y_{0}+\dot{\delta} \dot{y}_{0}+u_{1} \delta_{\tau_{1}}+u_{2} \delta_{\tau_{2}} .
\end{aligned}
$$

The cancellation of this term can be performed from (9) by means of simple multiplications an derivations w.r.t. $s$, yielding:

$$
\left[\frac{\partial^{5}}{\partial s^{5}}-\left(\tau_{1}+\tau_{2}\right) \frac{\partial^{4}}{\partial s^{4}}+\tau_{1} \tau_{2} \frac{\partial^{3}}{\partial s^{3}}\right] s \xi(s)=0
$$

Alternatively, Schwartz Theorem shows that $\alpha Q=0$ for any function $\alpha$ that vanishes on the support of $Q$, i.e. for any $\alpha$ satisfying $\alpha(0)=\dot{\alpha}(0)=\ddot{\alpha}(0)$, and $\alpha\left(\tau_{1}\right)=$ $\alpha\left(\tau_{2}\right)=0$. The choice $\alpha(t)=t^{3}\left(t-\tau_{1}\right)\left(t-\tau_{2}\right)$ finally results in (6), written explicitly:

$$
t^{3}\left(t-\tau_{1}\right)\left(t-\tau_{2}\right) \times\left[\left(\delta^{(3)}+a_{1} \delta^{(2)}+a_{0} \delta^{(1)}\right) * y\right]=0 .
$$

This latter equation is just a formulation of (11) in the time domain. In addition to providing a simpler method for the cancellation procedure, one of the main advantage of using a distributional approach lies in the ability to select arbitrary candidate functions $\alpha$ subject to local constraints at $0, \tau_{1}$ and $\tau_{2}$. As in Section 3.2 where bounded exponential functions have been used, such advantage allows us for instance to avoid multiplication of measurements with unbounded (polynomial) functions in case of noisy data. Note that, as mentioned above, the cancellation of $\xi$ did not eliminate the information about the delays, since they are recovered in the formulation of $\alpha$.

From the previous equation, it becomes clear that the estimation problem is linear if only the parameters $a_{i}$ are to be estimated. This aspect has already been studied (see e.g. [11,23], including the concept of weak iden- 
tifiability). In addition to the nonlinear structure, another specificity for the delays estimation is linked to the support of the entities derived from measurements. Section 3.1 considers the single delay identification problem, while Section 3.2 deals with simultaneous delay and parameters estimation. In Section 4 the problem of infinitely many delays is considered, and a local estimation is first proposed. Global estimators are next provided at the price of a change from non asymptotic (finite time) estimators to asymptotic ones. Unlike section 3, the estimation problem of this latter section is based on a two step procedure in which the estimated switching times are reintroduced in a parameter estimation algorithm.

\subsection{Integration by parts}

Taking advantage of polynomial or exponential functions, strictly causal relations (i.e. avoiding any measurement derivative) can be obtained by simple integrations. In the distribution framework, straightforward computations can be carried out using the Leibniz rule,

$$
\alpha \delta_{a}^{(n)}=\sum_{k=0}^{n}(-1)^{(n-k)} C_{n}^{k} \alpha^{(n-k)}(a) \delta_{a}^{(k)},
$$

combined with the following property involving convolution and multiplication (see e.g. [22]):

$$
\begin{aligned}
t^{n}(S * T) & =\sum_{k=0}^{n} C_{n}^{k}\left(t^{k} S\right) *\left(t^{n-k} T\right), \\
e^{-\gamma t}(S * T) & =e^{-\gamma t} S * e^{-\gamma t} T .
\end{aligned}
$$

Denoting $z_{i}=t^{i} y, z=e^{-\gamma t} y$ and $\lambda=e^{\gamma \tau}$, some simple manipulations based on $(13,14,15)$ permit to expand terms of the form $t^{p} y^{(q)}$ (resp. $e^{-\gamma t} y^{(q)}$ ) into sum of derivatives of $t^{k} y$ (resp. $e^{-\gamma t} y$ ), as shown in the examples below:

$$
\begin{aligned}
t^{3} y^{(2)} & =t^{3}\left(\delta^{(2)} * y\right)=-6 z_{1}+6 z_{2}^{(1)}-z_{3}^{(2)} \\
e^{-\gamma t} y^{(2)} & =e^{-\gamma t}\left(\delta^{(2)} * y\right)=\gamma^{2} z+2 \gamma z^{(1)}+z^{(2)}
\end{aligned}
$$

Therefore, successive integrations of these equations avoid any measurement derivative. It should be stressed that such integrations correspond to nothing but the well known integration by parts formula, although on a one hand, integrators may be replaced in a noisy context by any proper transfer function, and on the other hand, convolution with compact supported functions may be also considered for local estimation purposes. One of the main features of the presented approach consists in considering additional disturbances and/or perturbations as a sum of a constant term and a rapidly fluctuating signal with zero-mean. The first part is therefore straightforwardly annihilated by means of an additional derivation, while the effects of fluctuations are attenuated by the integrations or transfer functions mentioned above.
When facing noisy data, the selection of the candidate function $\alpha$ may affect the estimation results, since multiplying the measurements (and hence the noise) with $\alpha$ modifies the noise characteristics of the resulting equation. The integration by parts described in this section also aims at reducing these noise effects, but the design of an optimal pair ( $\alpha$, filter) is not a trivial task and requires further investigations. Practical implementations are shown (Figures 1,3).

\section{Application to delay systems identification}

Among the numerous open problems for delay systems, identification is most crucial [19]. On the one hand, various powerful control techniques (predictors, flatnessbased predictive control, finite spectrum assignments, observers, ...) may be applied if the dead-time is known. On the other hand, the existing identification techniques for time-delay systems (see, e.g., [17] for adaptive techniques or [18] for a modified least squares technique, and a survey in [5]) generally suffer from poor speed performance. To remain in a structured entries context, we focus on step (or piecewise constant input) responses, but the generalization to piecewise polynomial inputs is easy. Although in off-line applications the delay is usually estimated beforehand by looking at the delay between the step changes and the change in the measurements, this estimation can however become non trivial when facing noisy data, nonzero initial conditions, or when unknown parameters make difficult the distinction between the inertia of the process and the actual delay. This latter case where on-line simultaneous parameters and delay estimation is required is also studied in this section.

\subsection{Delay identification}

We consider here a first order nonlinear system subject to a delayed step input $u(t)=H(t-\tau)$. Such systems, where the delays only appear in the control variables, are most common in practice (see [9] for their theoretical background and their control), and this simple example emphasizes that linearity w.r.t. input-outputs is not required.

$$
\dot{y}+a \sin y=y_{0} \delta+\gamma_{0} H+b H(t-\tau),
$$

where $\gamma_{0}$ is a constant perturbation, $a, b$, and $\tau$ are constant parameters. Note that in the distribution sense, the initial condition term occurs as an impulsive term. The coefficient $a$ is assumed to be known for the moment. In the framework of Section 2, the structured term $\xi$ consists here in the right hand side member of (18) for which equation (3) is satisfied with:

$$
P=\dot{\delta}, \quad \text { and } \quad Q=y_{0} \dot{\delta}+\gamma_{0} \delta+b \delta_{\tau}
$$


In turn, equation (18) admits the equivalent description (5) with:

$$
R=(\dot{\delta}, a \delta), \quad \text { and } \quad \omega=(y, \sin y)^{T} .
$$

The distribution $Q$ is annihilated by any function $\alpha$ satisfying $\alpha(0)=\dot{\alpha}(0)=\alpha(\tau)=0$, yielding equation (6). Summing up for this simple example, a first order derivation results in equation (21) which may be canceled, for instance, by the polynomial $\alpha=t^{3}-\tau t^{2}$, yielding:

$$
\begin{aligned}
& \ddot{y}+a \dot{z}=y_{0} \dot{\delta}+\gamma_{0} \delta+b \delta_{\tau}, \\
& \left(t^{3}-\tau t^{2}\right)(\ddot{y}+a \dot{z})=0,
\end{aligned}
$$

where for ease of notation we have denoted $z=\sin y$. As an equality of distributions, equation (22) does not make sense for any $t$ (otherwise we would get from $(22) t=\tau$ ). More precisely, and from the r.h.s. of (21), it also reads:

$$
\begin{aligned}
& t^{3}(\ddot{y}+a \dot{z})=b t^{3} \delta_{\tau}=b \tau^{3} \delta_{\tau} \\
& t^{2}(\ddot{y}+a \dot{z})=b t^{2} \delta_{\tau}=b \tau^{2} \delta_{\tau}
\end{aligned}
$$

However, a single integration of (22) results in an equality of functions from which the delay becomes available. To ensure causality, $k \geq 2$ integrations can be used, yielding an explicit and non asymptotic formula of the delay:

$$
\tau=\frac{\int^{(k)} t^{3}(\ddot{y}+a \dot{z})}{\int^{(k)} t^{2}(\ddot{y}+a \dot{z})}, \quad t>\tau,
$$

where the symbol $\int^{(k)}$ stands for iterated integration of order $k$. Since from $(23,24), t^{q}(\ddot{y}+a \dot{z}), q=1,2$ have their support reduced to $\{\tau\}$, both numerator and denominator of $(25)$ are with support within $(\tau, \infty)$, so the delay is clearly not identifiable for $t<\tau$. Nevertheless, the delay estimation may be achieved in a small time interval $(\tau, \tau+\epsilon)$. According to the integration by parts of the previous section (see equation 16), Figure 1 is showing a partial realization scheme of the terms involved in (25).

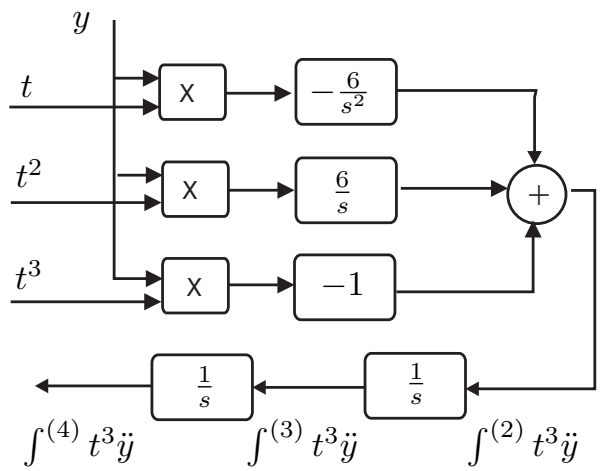

Fig. 1. Realization scheme of $\int^{(k)} t^{3} \ddot{y}, \quad k \geq 2$.

Simulation results in a noisy context are depicted in Figure 2, with $k=3$ and 4 integrations, $y(0)=-0.1$, $a=2.5, \tau=0.3$. Throughout this paper, simulated noise is obtained using uniformly distributed pseudo-random numbers generated by Matlab software. The signal-tonoise ratio, defined as $S N R=20 \log \left(\frac{y_{\max }-y_{\min }}{\sigma}\right)$, where $\sigma$ denotes the noise standard deviation, is fixed to $35 \mathrm{~dB}$. The choice of more than 2 integrations has been used to obtain an additional filtering effect which attenuates the noise from the measurement $y$.
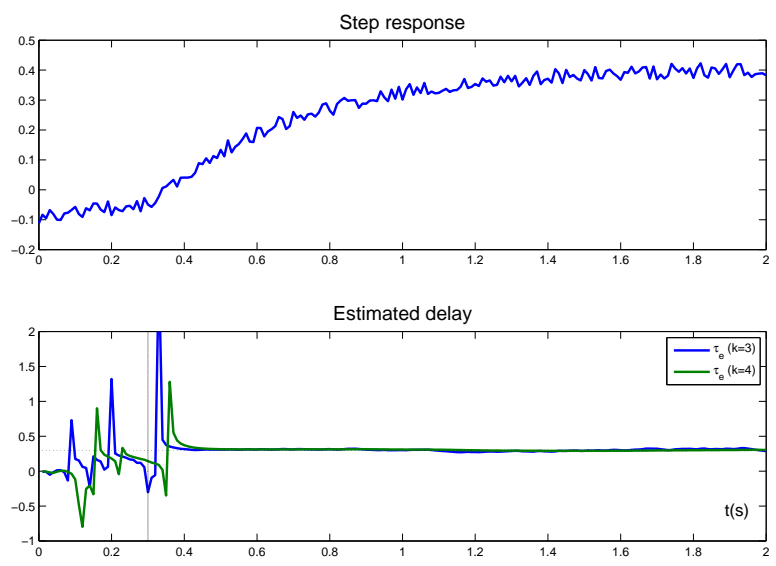

Fig. 2. Trajectory of (18) and delay identification.

The reliability of this non asymptotic approach has been tested on $N=100$ realizations and at different times estimation $t_{\text {est }}$, yielding the estimated means and standard deviations of Table 1.

\begin{tabular}{ccccc}
\hline$t_{\text {est }}$ & $2 \tau$ & $3 \tau$ & $4 \tau$ & $5 \tau$ \\
$\overline{\hat{\tau}}$ & 0.303 & 0.301 & 0.301 & 0.300 \\
$\sigma_{\hat{\tau}}$ & 0.014 & 0.005 & 0.005 & 0.006
\end{tabular}

Table 1

Mean values ans standard deviations of the estimated delay for $N=100$ realizations.

\subsection{Simultaneous parameters and delay identification and experimental results}

The aim of this paragraph is to show how the previous material can be used for a simultaneous identification of both parameters and delay. The developments are based on a first or second order process, although the generalization to higher order systems is straightforward. As in the previous example, the extension to a certain class of nonlinear systems is simple as well, provided the output derivatives $y^{(k)}$ of the linear framework are replaced by derivatives of known nonlinear functions $(g(y))^{(k)}$. Experiments are carried out on a Feedback process PT37 100. This process consists of heating the air flowing in a tube to the desired temperature level. The physical principle which governs the behavior of the this process is the balance of heat energy. The response of the sensor to a change in the heater power is moreover affected by 
a pure delay, which depends on the velocity of the process and the distance between the point of change and the sensor. In an open loop configuration, the behavior is approximated by a linear system with delayed input.

Two different models and experiments are considered in this section: (1) The process is subject to a step input, and a second order model

$$
G_{1}(s)=\frac{K e^{-\tau s}}{a_{2} s^{2}+a_{1} s+1},
$$

is used to describe it. The experiment starts from a rest position, allowing to neglect the initial conditions. (2) The process is subject to a piecewise constant input, and a simplified model (first order) is assumed, with nonzero initial conditions:

$$
G_{2}(s)=\frac{K e^{-\tau s}}{a s+1}
$$

\subsubsection{Second order model and step input}

The candidate function chosen for the annihilation of the structured part is the complex and bounded function $\alpha=\left(1-\lambda e^{-j \omega t}\right)$, with the unknown to be found $\lambda=$ $e^{j \omega \tau} \in \mathbb{C}$, and tunable frequency $\omega$. More explicitly, a derivation of the differential equation derived from (26), followed by the multiplication by $\alpha$ result respectively in:

$$
\begin{aligned}
& a_{2} y^{(3)}+a_{1} y^{(2)}+y^{(1)}=K \delta_{\tau}, \\
& \left(1-\lambda e^{-j \omega t}\right)\left(a_{2} y^{(3)}+a_{1} y^{(2)}+y^{(1)}\right)=0 .
\end{aligned}
$$

We shall focus on the identification of the coefficients $\left\{\lambda, a_{2}, a_{1}\right\}$, and provided a sufficiently large period $2 \pi / \omega$, the delay is deduced from the unique argument $\tau=$ $\arg (\lambda) / \omega$. Due to the terms $\lambda a_{i}, i=1,2,(29)$ is not linear w.r.t. the unknown coefficients, but may be written in the following form:

$$
\left[\left(y^{(3)}, \cdots, y^{(1)}\right)-\lambda\left(e y^{(3)}, \cdots, e y^{(1)}\right)\right]\left(\begin{array}{c}
a_{2} \\
a_{1} \\
1
\end{array}\right)=0
$$

where for ease of notation, we have denoted $e=e^{-j \omega t}$. As in the previous section, successive integrations transform the equality of singular distributions of (29) into one of continuous functions. However, denoting $\Theta=$ $\left(a_{2}, a_{1}, 1\right)^{T}$ the (normalized) vector of parameters, the specific structure of (30) leads to following generalized eigenvalue problem for possibly non square pencils:

$$
(A-\lambda B) \Theta=0
$$

where, using a Matlab-like notation, the entries of the $m \times 3$ trajectory-dependent matrices $A$ and $B$ are given by

$$
\begin{aligned}
A(i,:) & =\int^{(i+2)}\left(y^{(3)}, \cdots, y^{(1)}\right), \quad i=1, \ldots, m, \\
B(i,:) & =\int^{(i+2)}\left(e y^{(3)}, \cdots, e y^{(1)}\right) \quad i=1, \ldots, m .
\end{aligned}
$$

The implementation of $B(i, j)$ is carried out according to the integration by parts formulas of the previous section. Recalling the Laplace transform property $\mathcal{L}\left(e^{-\gamma t} \dot{y}\right)=(s+\gamma) y(s+\gamma)$, these implementations also admit complex realization schemes, as illustrated below.

$$
\mathcal{L}\left(\int^{(4)} e^{-\gamma t} \ddot{y}\right)=\frac{(s+\gamma)^{2}}{s^{4}} y(s+\gamma),
$$

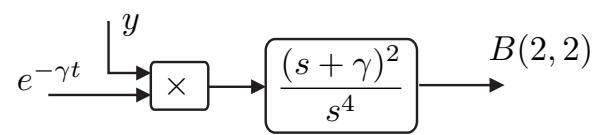

Fig. 3. Realisation scheme of $B(2,2)$.

Therefore, the identification problem has been transformed into the eigenvalue problem (31) in which, at each $t$, the unknown delay $\tau=\arg (\lambda) / \omega$ is derived from one eigenvalue, while the parameters $a_{1}, a_{2}$ are obtained from the corresponding normalized eigenvector. Solving (31) in a noisy context and in the non-square case (i.e. by considering $m>3$ lines for $A$ and $B$ ) is generally not an easy task, since (31) "has the awkward feature that most matrices have no eigenvalues at all, whilst for those that do, an infinitesimal perturbation will in general remove them" [26]. A possible approach can be based on the pseudo-spectra analysis which consists in defining the $\epsilon$-pseudospectra of (31) as the set

$$
\Lambda_{\epsilon}=\left\{z \in \mathbb{C}:\|A+z B\|^{+}>\epsilon^{-1}\right\} .
$$

(see e.g [26] and the references therein). However, this approach is not appropriate for on-line perspectives, and we adopt here a simpler technique, based on the a priori stationarity assumption of the unknown parameters. More precisely, the selected parameters correspond to the eigenpair $\left(\lambda_{i}, \Theta_{i}\right)$ of the square pencil (31) that minimizes the norm $\left\|\left(A+\lambda_{i} B\right) \Theta_{i}\right\|$ of the rectangular pencil (i.e. for $m>3$ ).

As in the single delay estimation problem of the previous section, it can be easily shown that matrices $A$ and $B$ are continuous matrix functions with support within $(\tau, \infty)$, which means that the delay and parameters are not identifiable for $t<\tau$. Moreover, and although the 
approach is non asymptotic, this continuity can make the estimation problem sensitive to noise and neglected dynamics in the vicinity of $\tau$. Unlike noise-free contexts or reduced order identification problems such as the delay estimation in section 3.1, it is clear a minimum amount of trajectories information is required here to obtain a consistent and relevant eigenvalue problem. Moreover, the integrations involved in equation (32) show that at each time $t$ the estimation algorithm is based on the whole available data (from 0 to $t$ ). Hence even at its steadystate phase, the eigenvalue problem is still consistent.

Figure 4 (top) shows the experimental response as well as the simulated trajectories based on the identified delay and parameters (bottom). Although the convergence algorithm is clear, the time history of the identified parameters reflects a singularity of the eigenvalue problem in the vicinity of $t=0.8 \mathrm{~s}$. The implementation of (32) was converted to discrete-time, assuming a sampling period of 50 msec. Resolution of (31) has been made using the polyeig function of the Matlab software.
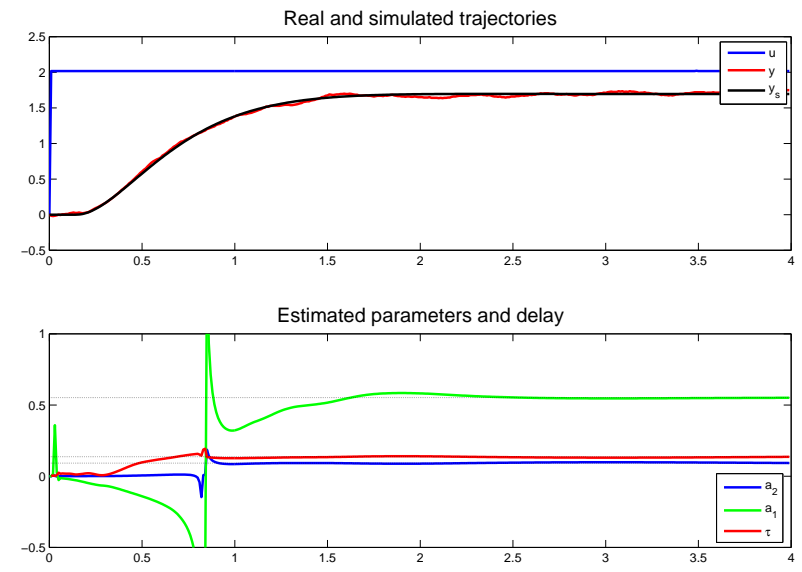

Fig. 4. Experimental and simulated trajectories (top). Estimated delay and parameters (bottom)

Taking into account the static gain $K$ estimated by other means, the identified second order model for the process reads:

$$
G_{1}(s) \approx \frac{0.84 e^{-0.13 s}}{0.09 s^{2}+0.55 s+1} .
$$

\subsubsection{First order model and piecewise constant input}

This paragraph aims at proving the robustness of the estimation approach in case of neglected dynamics in modeling. In this experiment, a simplified first order model of equation (27) is adopted, and the process is subject to the structured input consisting in a piecewise constant signal with equally spaced discontinuities,

$$
u(t)=\sum_{k=0}^{\infty} u_{k} \chi_{[k T,(k+1) T]}(t),
$$

where $\chi_{[X]}(t)$ denotes the characteristic function of the set $X$, and $T>0$. Taking into account the nonzero initial condition, an input/output description of the process and a first order derivation yield respectively:

$y+a \dot{y}=y_{0} \delta+K \delta_{\tau} * u$,
$\dot{y}+a \ddot{y}=y_{0} \dot{\delta}+K \sum_{k=0}^{\infty} \sigma_{k} \delta_{\tau+k T}$,

where $\sigma_{k}$ denotes the jumps $K\left(u_{k}-u_{k-1}\right)$, of the right hand side member, with $u_{-1}=0$. Now according to Schwartz Theorem, multiplication of (38) by any function $\alpha$ satisfying $\dot{\alpha}(0)=\alpha(0)=\alpha(\tau+k T)=0$ annihilate the r.h.s., and the following cancellation is proposed:

$$
\left(1-e^{-\gamma t}\right)^{2}\left(1-\lambda e^{-j \omega t}\right)(\ddot{y}+a \dot{y})=0,
$$

for some $\gamma>0$ and $\omega=2 \pi / T$. Here again, the formed relation no longer depends on initial condition nor on the magnitude of the jumps. For the sake of concision, we let $\alpha_{1}=\left(1-e^{-\gamma t}\right)^{2}, \alpha_{2}=e^{-j \omega t} \alpha_{1}$, and rewrite (39) in the form:

$$
\left[\left(\begin{array}{ll}
\alpha_{1} \ddot{y} & \alpha_{1} \dot{y}
\end{array}\right)-\lambda\left(\begin{array}{ll}
\alpha_{2} \ddot{y} & \alpha_{2} \dot{y}
\end{array}\right)\right]\left(\begin{array}{l}
a \\
1
\end{array}\right)=0 .
$$

We recover here the same (but reduced) structure of the previous second order model. Successive integrations lead to an eigenvalue problem, solved following the former approach. Experimental and simulated trajectories, as well as time history of the estimates $a$ and $\tau$ are depicted in Figure 5. The estimated model reads in this case:

$$
G_{2}(s) \approx \frac{0.84 e^{-0.26 s}}{0.43 s+1} .
$$
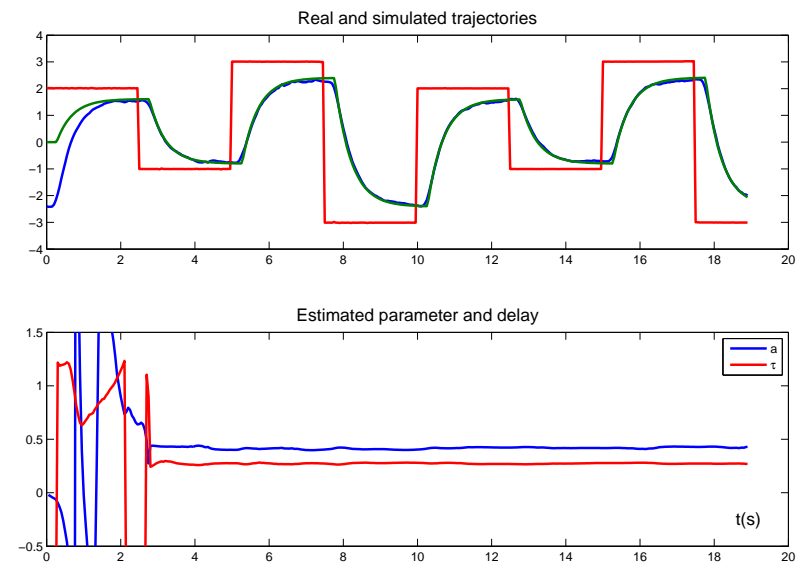

Fig. 5. Experimental and simulated trajectories (top). Estimated delay and parameter (bottom)

Note that the estimated delay is larger than that obtained for the second order model, and a zoom in on the 
vicinity of a step change can shown lower performances compared to the second order model. However, although this value does not exactly reflect the physical delay of the process, combined with the estimation of $a$, it compensates the smoothing effect (inertia) of the neglected second order dynamic in the vicinity of a step change.

\section{Application to a simple switching process}

Many works have been devoted to state estimation of hybrid systems (see e.g. $[15,21,14]$ ), while parameters estimation, in case of piecewise affine systems, is rather viewed as the problem of reconstructing the piecewise affine map $[12,20]$. In all cases, these techniques are based on a probabilistic setting (particle filtering, clustering or classification). Based on the former technique, this section aims at suggesting new tools that can be viewed as a first step toward a more general approach for a class of switched systems. The process under consideration is described by the switched linear system:

$$
\dot{y}+a y=k u, \quad a(t)=\sum_{i} a_{i} \chi_{\left[\tau_{i}, \tau_{i+1}\right]}(t),
$$

where $\tau_{0}=0<\tau_{1}<\tau_{2} \ldots$ denote the switching times and where $\chi_{[X]}(t)$ the characteristic function of the set $X$. Note that the switching rule (external or state dependent) is not taken into account here. The gain $k$ is assumed to be known and we shall focus on the on-line estimation of both the switching times and the discontinuous function $a$. Let us first observe that in case of an estimation of the restriction of $a$ to any interval $\left(t_{0}, t\right)$ that does not contain switch times, we recover the linear case already treated [11]. In such case, a multiplication of (42) by $t$ which cancels the possible nonzero initial condition term, followed by an integration by part easily results in the non asymptotic estimation:

$$
\begin{gathered}
\hat{a}_{\mid\left(t_{0}, t\right)}=\frac{k \int_{t_{0}}^{t} \theta u d \theta-t y+t_{0} y\left(t_{0}\right)+\int_{t_{0}}^{t} y d \theta}{\int_{t_{0}}^{t} \theta y d \theta} \\
\left(t_{0}, t\right) \not \supset \tau_{i}, \quad i=1,2, \cdots
\end{gathered}
$$

This estimation can be extended to $\mathbb{R}_{+} \backslash\left\{\tau_{i}\right\}$ if the lower bound $t_{0}$ is replaced by any function $\tilde{b}(t)$ satisfying:

$$
b(t) \leq \tilde{b}(t)<t, \quad \text { with } \quad b(t)=\sum_{i} \tau_{i} \chi_{\left[\tau_{i}, \tau_{i+1}\right]}(t)
$$

yielding the estimate of the time varying parameter:

$$
\hat{a}=\frac{k \int_{\tilde{b}(t)}^{t} \theta u d \theta-t y+\tilde{b}(t) y(\tilde{b}(t))+\int_{\tilde{b}(t)}^{t} y d \theta}{\int_{\tilde{b}(t)}^{t} \theta y d \theta}
$$

Therefore, the remaining task will consist in the sequel in providing an estimate $\hat{b}$ of the switching function $b$ given in (44). The identification algorithm (45) will be based on this estimation. In order to avoid additional singularities and indetermination at 0 , we shall assume that $u$ is continuous and $y$ is positive. We therefore get from (42):

$$
a=k \frac{u}{y}-\frac{\dot{y}}{y}=k z-\dot{w}, \quad z:=u / y, \quad w:=\log (y)
$$

As a piece wise constant function, $a$ admits the following singular derivative:

$$
\dot{a}=k \dot{z}-\ddot{w}=\sum_{i} \sigma_{i} \delta_{\tau_{i}}
$$

where the $\sigma_{i} i=1,2 \cdots$ denote the unknown jumps of $a$ at $\tau_{i}$, while $\sigma_{0}$ contains possible additional terms due to the jumps of $z$ and $w$ at 0 . The estimation principle for the commutation times is not quite different from that of the delays, since it is based on multiplications followed by integrations by parts. However, the former iterated integrals $\left(\int^{(k)}\right)$, corresponding to iterated convolution with a Heaviside function, will be replaced by a convolution with a more general term $\alpha$. Let us denote:

$$
z_{0}=z, z_{i}=t^{i} z, \quad w_{0}=w, w_{i}=t^{i} w .
$$

Recalling the properties $\dot{\alpha} * x=\alpha * \dot{x}, t^{k} \delta_{\tau}=\tau^{k} \delta_{\tau}$, and using the expanding formulation $(14,16)$ of the introductory section, some simple manipulations yield from (47):

$$
\begin{aligned}
\alpha *(t \dot{a}) & =\dot{\alpha} * z_{1}-\alpha * z-\ddot{\alpha} * w_{1}+2 \dot{\alpha} * w \\
& =\sum_{i} \sigma_{i} \tau_{i}\left(\delta_{\tau_{i}} * \alpha\right), \\
\alpha *\left(t^{2} \dot{a}\right) & =\dot{\alpha} * z_{2}-2 \alpha * z_{1}-\ddot{\alpha} * w_{2}+4 \dot{\alpha} * w_{1}-2 \alpha * w_{0} \\
& =\sum_{i} \sigma_{i} \tau_{i}^{2}\left(\delta_{\tau_{i}} * \alpha\right) .
\end{aligned}
$$

The causality requirement implies here that the selected function $\alpha$ is twice differentiable with support $\subset(0, \infty)$. For such $\alpha$, and although the jumps $\sigma_{i}$ of $a$ are by nature unknown, the left hand side members of these equations are available quantities whose manipulation will allow the switching times estimation. Whether we are facing one or multiple switches, there will be a notable difference in the design of the estimation algorithms. Moreover, it is worth noticing that the proposed estimation technique is a two-step procedure in which the commutation times are estimated first and then introduced in the identification algorithm (45). In this context, other estimation or filtering algorithms can exploit the switch time information provided by the first step.

\subsection{The single switch case}

Such situations may be of interest in many applications such as in food industry where first order linear models 
are used to describe dying food processes and two-step drying (pre-drying and drying at higher temperature) are performed on fruits to extract the water out of the product while preserving its qualities of interest [25]. The commutation function to be estimated reads in this case $b(t)=\tau_{1} H\left(t-\tau_{1}\right)$, and equations $(49,50)$ reduce to:

$$
\begin{aligned}
\alpha *(t \dot{a}) & :=n(t)=\sigma_{1} \tau_{1} \alpha\left(t-\tau_{1}\right), \\
\alpha *\left(t^{2} \dot{a}\right) & :=d(t)=\sigma_{1} \tau_{1}^{2} \alpha\left(t-\tau_{1}\right) .
\end{aligned}
$$

A direct approach for the estimation of $\tau_{1}$ would consist in forming the ratio $\tau_{1}=\frac{d(t)}{n(t)}$ for all nonzero values of $d(t)$. Nevertheless, this simple procedure raises the problem of the indetermination of the ratio, particularly for $t \leq \tau_{1}$ (since $\left.\operatorname{supp} n, d \subset\left(\tau_{1}, \infty\right)\right)$. To avoid such indetermination and the use of some fixed and a priori threshold, the following asymptotic algorithm is proposed:

$$
\dot{\hat{b}}=-|n(t)| \hat{b}+|d(t)|, \quad b(0)=\tau_{0}(=0)
$$

An explicit solution of this differential equation can be easily obtained using standard mathematical tools (i.e. homogeneous solution $k e^{-\int_{0}^{t}|n(\theta)| d \theta}$, and variation of constant formula). Taking advantage of the relation $d(t)=\tau_{1} n(t)$, it is readily seen that this estimator admits the explicit form:

$$
\hat{b}(t)=\tau_{0} e^{-\int_{0}^{t}|n(\theta)| d \theta}+\tau_{1}\left(1-e^{-\int_{0}^{t}|n(\theta)| d \theta}\right) .
$$

Since $n(t)=0$ on $\left(0, \tau_{1}\right), \hat{b}$ is first maintained at its initial value $\tau_{0}$, and provided $\int_{0}^{t}|\alpha(\theta)| d \theta \rightarrow \infty$ as $t \rightarrow \infty$, the estimator will next converge asymptotically to the desired switch time $\tau_{1}$. It is worthy of note that from (51), the dependence of $n$ with respect to the unknown jump $\sigma_{1}$ makes the rate of convergence uncontrollable, but this rate can yet be modified using a tunable gain $K$ in the design of $\alpha$. Note that in a noisy context, the non linearities in the estimation algorithm of $b$ may introduce bias in the switching time estimates. However, it is worthy of note that these noise effects do not directly affect the estimation of the time-varying coefficient $a$. This is not a surprising result if one recalls that this estimation, described by algorithm (45), does not require the exact values of the switching times, but only a lower bound $\tilde{b}(t)$ satisfying (44).

Simulation results in a noisy context are shown in Figure 6 , with a SNR of $37 \mathrm{~dB}$ for both input and output. The coefficient $a$ of this example occurs a jump from 0.4 to 0.8 at $t=8 s$. The input is given by $u=0.3(2+\cos (0.5 t)(1+$ $\sin (0.2 t))$ ), and the implementation was converted to discrete-time with a sampling period of $50 \mathrm{msec}$. The selected function $\alpha$ for the realization of $(51,52)$ is given by:

$$
\alpha(s)=\frac{10}{s(s+1)^{3}} .
$$

Figure 6 (center) shows the time history of the algorithm (53). This switch time estimate is used for the identification of $a$ in(45), yielding the response depicted in Figure 6 (bottom).

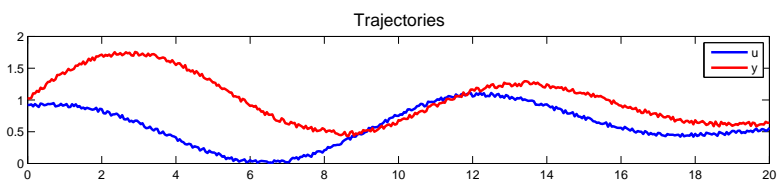

Simulated and estimated switch time

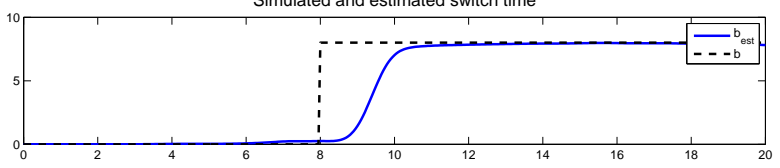

Simulated and estimated time varying parameter a

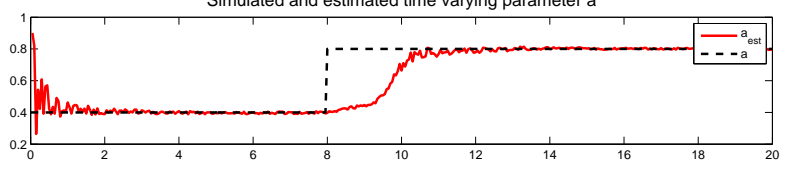

Fig. 6. Simulated trajectories (top). Simulated and estimated switch function $b$ (center). Simulated and estimated time varying parameter $a$ (bottom)

Although the proposed method aims at forming a continuous time estimation of the time-varying parameter $a$, the mean values of the estimated switching times, computed at $t=(8,20)$ and for $N=100$ realizations, are $(\overline{\hat{b}}(8), \overline{\hat{b}}(20))=(0.030,8.43)$, while the mean and standard deviation of the estimated parameter $a$ are respectively $(\overline{\hat{a}}(8), \overline{\hat{a}}(20))=(0.400,0.800)$, and $\left(\sigma_{a}(8), \sigma_{a}(20)\right)=(0.005,0.003)$. As mentioned above, these results confirm that the possible bias in the switching times estimation does not affect the accuracy of the estimated parameter $a$.

\subsection{Estimation with multiple switches}

The specificity of the estimation problem for the general case lies in the fact that in forming the derivative $\dot{a}$ in (47), neither the jumps $\sigma_{i}$ nor the commutation times $\tau_{i}$ are known. Unlike the previous single switch case where the ratio $d(t) / n(t)$ was allowing the jump cancellation regardless of the choice of the convolution term $\alpha$, this procedure requires more refinement for multiple switches. This ratio, when defined, reads:

$$
\frac{d(t)}{n(t)}=\frac{\alpha *\left(t^{2} \dot{a}\right)}{\alpha *(t \dot{a})}=\frac{\sum \sigma_{i} \tau_{i}^{2} \alpha\left(t-\tau_{i}\right)}{\sum \sigma_{i} \tau_{i} \alpha\left(t-\tau_{i}\right)}
$$

In this relation, the jumps cancellation still holds, at least locally, if the candidate function $\alpha$ has an appropriate compact support. To ensure the algorithm convergence, let us further assume significant changes in the unknown coefficient $a(t)$ in terms of magnitude and/or duration. More precisely, we assumes that the jumps are 
of significant magnitude, and that we are given a lower bound for the delay between two successive commutations:

$$
\text { (a) } \sigma_{i} \geq \sigma_{\min }, \quad \text { (b) } \tau_{i}-\tau_{i-1} \geq \Delta, \quad i=1,2 \cdots \text {. }
$$

By considering a smooth function $\alpha$ with support within $(0, \Delta)$, one therefore gets from (56) the non asymptotic but local switches identification algorithm:

$$
\tau_{i}=\frac{d(t)}{n(t)}, \quad t \in\left(\tau_{i}, \tau_{i}+\Delta\right)
$$

The selected $\alpha$ for this application is given by $\sin ^{4}(\pi t / \Delta)$ and is shown, with its derivatives, on Figure 7 (top). The resulting functions $n(t)$ and $d(t)$ are depicted in Figure 7(bottom) for a given input/output pair shown in Figure 8(top). Here again, and although the simple ra-
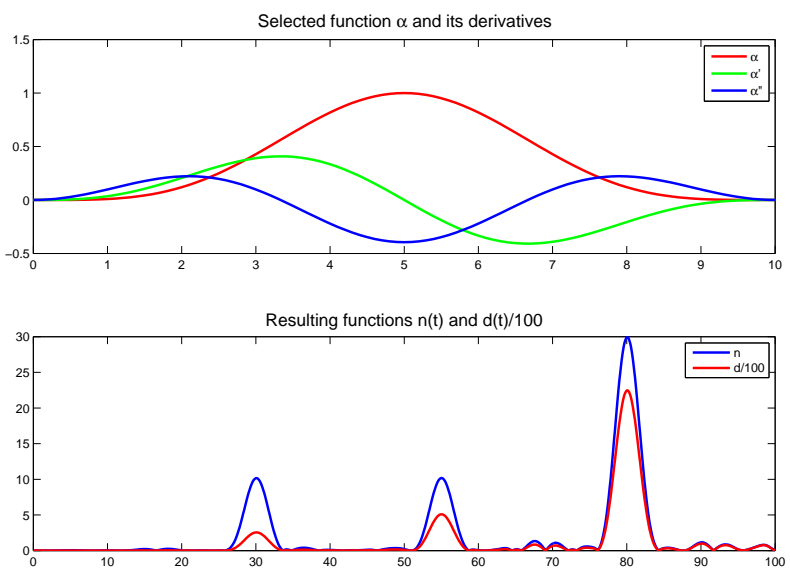

Fig. 7. Selected function $\alpha$ (top). Resulting function $n(t)$ and $d(t)$ in a noisy context(bottom).

tio (58) may be sufficient for off-line applications, we can use the on-line algorithm (53) based on the new entries $n$ and $d$, and in which neither a priori thresholds nor initialization procedures are required. Since $n$ and $d$ are zero on $\left(\tau_{i}+\Delta, \tau_{i+1}\right)$, the estimator response is governed by the same equation as in (54), except that the convergence is required on the limited interval $\left(\tau_{i}, \tau_{i}+\Delta\right)$. Condition (57a) is used to ensure this convergence. More precisely, denoting $S=\int_{0}^{\Delta} \alpha(\theta) d \theta$, the terms $\int_{\tau_{i}}^{\tau_{i}+\Delta}|n(\theta)| d \theta=\tau_{i}\left|\sigma_{i}\right| S$ in (54) are expected to take sufficiently large values. However, since $\left\{\tau_{i}\right\}$ is an increasing sequence, practical limitations imposed by these constraints mainly concern the first commutations.

Figure 8(top) show the input/output trajectories of the plant subject to an input $u=0.3(5+\cos (0.35 t)(2+$ $\sin (0.13 t))$ ) an a time varying coefficient $a$ given in (42) with $\left\{a_{i}\right\}=\{0.6,1, .8,1.2, .6\}$ and $\left\{\tau_{i}\right\}=$ $\{0,30,55,80,100\} s$. The SNR is fixed to $45 \mathrm{~dB}$ for both input and output signals, the discretization step is
$100 \mathrm{~ms}$, and the convolution products are performed using simple sums. Figure 8(center) shows the time history of the switching time estimator $\hat{b}(t)$ in both free and noisy context, as well as the simulated switch function $b(t)=\sum_{i} \tau_{i} \chi_{\left[\tau_{i}, \tau_{i+1}\right]}(t)$, and the reference time $t$.(dashed). The simulated (dashed) and estimated coefficient $a$ in the noisy context are depicted in Figure 8(bottom).
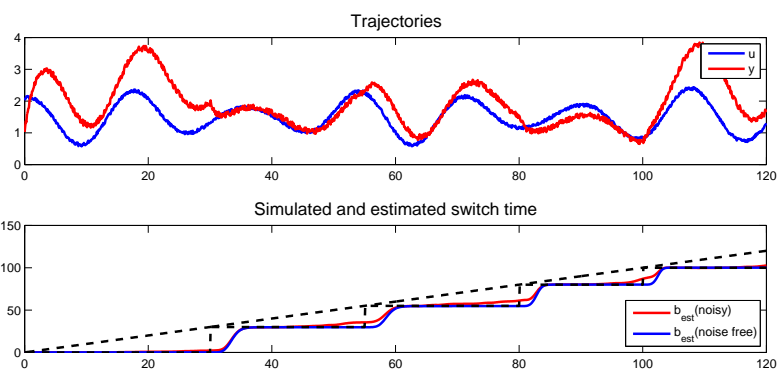

Simulated and estimated time varying parameter a

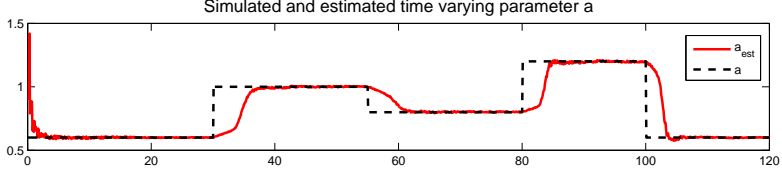

Fig. 8. Trajectories(top). Switching time estimator $\hat{b}$ in both noise free and noisy context (center). Simulated and estimated time varying parameter $a$ (bottom)

Statistical results at the switching times are given in Table 2. Here again, satisfactory estimates of the parameter $a$ are obtained despite the deviations in the switching times estimates.

\begin{tabular}{cccccc}
\hline$\overline{\hat{b}}$ & 8.69 & 45.3 & 69.5 & 92.4 & 110.1 \\
$\overline{\hat{a}}$ & 0.600 & 1.000 & 0.799 & 1.199 & 0.600 \\
$\sigma_{\hat{a}}$ & 0.001 & 0.006 & 0.004 & 0.013 & 0.004 \\
\hline
\end{tabular}

Table 2

Mean values of the estimated switching times $\hat{b}$ and parameter $\hat{a}$, and standard deviation of $\hat{a}$ at the simulated switching times $t=(30,50,80,100)$ and for $N=100$ realizations.

\section{Conclusion}

This paper has presented new tools for the parameters estimation of systems involving retarded phenomena. In continuous-time identification, delays and switching parameters estimations are often considered as two different problems and different approaches are taken for the two purposes. In this work, a unified method has been proposed using simple algebraic techniques based on annihilation and integration. The performance of the proposed procedure is demonstrated by experimental applications. The ability to estimate parameters and delays using non asymptotic methods may provide new perspectives for real time control procedures. 


\section{References}

[1] S. Ahmed, B. Huang, and S.L. Shah. Parameter and delay estimation of continuous-time models using a linear filter. Journal of Process Control, 16:323-331, 2006.

[2] L. Belkoura. Identifiability of systems described by convolution equations. Automatica, 41:505-512, 2005.

[3] L. Belkoura and J.-P. Richard. A distribution framework for the fast identification of linear systems with delays. 6th IFAC Workshop on Time Delay Systems, TDS06, L Aquila, Italy, July 10-12, 2006.

[4] L. Belkoura, J.-P. Richard, and M. Fliess. On-line identification of systems with delayed inputs. 16th Conf. Mathematical Theory of Networks \& Systems, MTNS 06, Kyoto, Japan, July, 2006.

[5] Orlov Y. Richard J-P Belkoura L., Dambrine M. Identifiability and identification of linear systems with delays. Springer, LNCSE Advances in Time Delay Systems, 38:123135, 2004.

[6] F. Beltran-Carvajal, G. Silva-Navarro, H. Sira-Ramirez, and J. Quezada-Andrade. Active vibration control using on-line algebraic identification of harmonic vibrations. Proc. Amer. Control Conf, Portland, OR, 2005.

[7] M. Fliess, C. Join, and H. Sira-Ramirez. Robust residual generation for linear fault diagnosis: an algebraic setting with examples. Internat. J. Control, 77:1223-1242, 2004.

[8] M. Fliess, C. Join, and H. Sira-Ramirez. Non-linear estimation is easy. Int. J. Modelling, Identification and Control, vol 3, 2008.

[9] M. Fliess, R. Marquez, and H. Mounier. An extension of predictive control, pid regulators and smith predictors to some linear delay systems. Internat. J. Control, 75:728-743, 2002 .

[10] M. Fliess, M. Mboup, H. Mounier, and H. Sira-Ramirez. Questioning some paradigms of signal processing via concrete examples. Algebraic Methods in Flatness, Signal Processing and State Estimation, Editiorial Lagares, Mexico, pages 1$21,2003$.

[11] Sira-Ramirez H. Fliess M. An algebraic framework for linear identification. ESAIM Control, Optimization ans Calculus of Variations, 9, 2003.

[12] Ferrari-Trecate G., Muselli M., Liberaty D., and Morari M. Identification of piecewise affine and hybrid systems. Amercican Control Conference, Arlington US, pages 35213526, June 2001.

[13] O. Gomez, Y. Orlov, and IV. Kolmanovsky. On-line identification of siso linear time-invariant delay systems from output measurements. Automatica, 43:2060-2069, 2007.

[14] X Hulhoven, A. Vande Wouwer, and Ph. Bogarts. Hybrid extended Luenberger-asymptotic observer for bioprocess state estimation. Chemical engineering science, vol 61, 2006.

[15] X.D. Koutsouos. Estimation of hybrid systems using discrete sensors. 42nd Conference on Decision and Control, Hawaii, USA, 2003.

[16] Mamadou Mboup, Cdric Join, and Michel Fliess. An on-line change-point detection method. 16th Mediterrean Conference on Control and Automation, June, Ajaccio, France, 2008.

[17] Y. Orlov, I.V. Kolmanovsky, and O. Gomez. On-line identification of siso linear time-delay systems from output measurements: Theory and applications to engine transient fuel identification. Proceedings of the 2006 American Control Conference Minneapolis, Minnesota, USA, June 14-16, 2006.
[18] X.M. Ren, A.B. Rad, P.T. Chan, and W.L. Lo. On line identification of continuous-time systems with unknown time delay. IEE Trans. Aut. Control, 50-9:1418-1422, 2005.

[19] JP. Richard. Time-delay systems: an overview of some recent advances and open problems. Automatica, 39:1667-1694, 2003.

[20] Druhle S., Ferrari-Trecate G., de Jong H., and Viari A. Reconstruction of switching thresholds in piecewise-affine models of genetic regulatory networks. 9th Int. Workshop, HSCC, Amercican Control Conference, Arlington US, pages 184-199, 2006.

[21] E. De Santis and MD. Di Benedetto. Design of luenberger-like observers for detectable switching systems. Proc. of the 2005 IEEE International Symposium on Mediterrean Conference on Control and Automation, 2005.

[22] L. Schwartz. Théorie des distributions. Hermann, Paris, 1966.

[23] H. Sira-Ramirez. Some applications of differential algebra in systems identification. 3rd IFAC Symposium on System, Structure and Control, Brazil, October 2007, Plenary lecture.

[24] F. Veysset, L. Belkoura, P. Coton, and J-P. Richard. Delay system identification applied to the longitudinal flight of an aircraft through a verttical gust. 14th IEEE Mediterranean Conf. on Control and Automation, 2006.

[25] Nuninger W., Catiau L., and Montastruc L. Online optimisation of fruit drying based on drying curve identification. Socit Franaise de Gnie des Procds, St Etienne, 9-11 October, 2007.

[26] T.G. Wright and L.N. Trefethen. Eigenvalues and pseudospectra of rectangular matrices. Tech. Report, Oxford University, Numerical Analysis Group, 2001. 